\title{
ENERGY-DISPERSIVE RESIDUAL STRESS ANALYSIS UNDER LABORATORY CONDITIONS: CONCEPT FOR A NEW TYPE OF DIFFRACTOMETER
}

\author{
A. Liehr ${ }^{1, a}$, M. Klaus ${ }^{2, b}$, W. Zinn ${ }^{1, c}$, Ch. Genzel ${ }^{2, d}$ and B. Scholtes ${ }^{1, e}$
}

${ }^{1}$ Institute of Materials Engineering, University of Kassel, Mönchebergstr. 3, 34125 Kassel, Germany

\author{
${ }^{2}$ Helmholtz-Zentrum Berlin of Functional Materials, \\ Department Microstructure and Residual Stress Analysis, Albert-Einstein-Straße 15, \\ 12489 Berlin, Germany, klaus@helmholtz-berlin.de \\ aliehr@uni-kassel.de, bklaus@helmholtz-berlin.de, 'cinn@uni-kassel.de, \\ 'genzel@helmholtz-berlin.de, ${ }^{\mathrm{e}}$ scholtes@uni-kassel.de
}

Keywords: Energy-dispersive diffraction, laboratory X-ray source, residual stress depth profiling, information depth, instrumental resolution.

\begin{abstract}
In the past decade energy-dispersive (ED) synchrotron diffraction has evolved into a powerful tool for materials analysis. Recording complete diffraction patterns in rather few different measuring directions allows for depth-resolved analysis not only of the near-surface residual stress state, but also of composition and even texture gradients. However, since the number of synchrotron beamlines dedicated to ED-diffraction is restricted to very few instruments, alternatives have to be found which allow for ED residual stress analysis even under low flux laboratory conditions. In this project we start to establish the scientific basis for a measuring and evaluation method to make the transfer of the ED method to the laboratory dimensions possible, which is adapted to the conditions of much lower photon flux and larger beam divergences of laboratory X-ray sources. In this paper, we present the concept of an ED-diffractometer which is equipped with two detectors to enable simultaneous data acquisition for two orientations of the diffraction vector with respect to the sample reference system. The first constructive and experimental steps are presented and furthermore the possibilities and limitations of the new laboratory method and the advantages of the ED diffraction method to realize short measurement times in order to realize a high resolution of information depth are discussed.
\end{abstract}

\section{Introduction}

The key advantage of the energy dispersive diffraction method is the possibility to obtain full diffraction spectra in short measuring times under fixed but user defined scattering angles, which also contain the fluorescence lines of the chemical elements involved in addition to the interference $\mathrm{E}^{\mathrm{hkl}}$ from the crystalline phases of the investigated microstructure. The first reports about energy dispersive X-ray stress analysis (ED XSA) were published in the late 70s [1] and in the early 80s [2, 3 ] of the last century. Since then, numerous studies have been published which deal with methodological developments in the field of ED XSA using the main benefits of the ED diffraction mode. A series of publications from the early years of the ED diffraction method also deals with questions of instrumental resolution $[4,5]$, which still is a key factor for the application of this method. In only relatively few papers the white beam technique in reflection geometry for depth resolved residual stress analysis in the subsurface layer region is used. In this regard, pioneering work was supported by the universal plot method (UVP) applied to the case of ED diffraction [6]. In $[7,8]$, the bremsstrahlung of a conventional tungsten laboratory X-ray source was used in the early 90 s to demonstrate on a polished steel plate, that the values determined from different 
diffraction interference $\mathrm{E}^{\mathrm{hkl}}$ are assigned to different levels of information depth and can fit together to one master curve yielding a complete residual stress depth profile. The idea of a systematic transfer of the angle dispersive X-ray stress analysis (AD XSA) methods for the determination of near surface residual stress gradients to the ED case was taken up ten years later and realized in a laboratory scale in $[9,10]$. A further approach with a specific focus on thin film applications can be found for example in [11]. In [12] it has been shown recently, that the ED XSA is also working in the case of a conventional tungsten anode to determine a very steep stress gradient in good agreement with corresponding synchrotron measurements. Numerous stress analyses exist using the ED-XSA in reflection geometry to analyze mechanically surface treated materials. The achievable information depths, depending on the absorption of the used material, lay between some 10 microns and a few 100 microns. The intermediate depth range between the surface and the sample volume can be detected in this way without using the layer removal method.

Altogether, a lack of systematic studies and of a scientific basis with adapted measurement strategies for fast and reliable ED-residual stress analyses can be stated. In addition, the necessary specific laboratory equipment is missing, which is particularly due to the fact that up to now the corresponding experiments were mostly performed with makeshift constructions. A diffractometer specifically adapted for the demands of ED stress and texture measurements considering in its technical design recent experiences as mentioned above does currently not exist. This underscores the need for research in this field of measurement methods.

\section{Concept of hardware}

The energy dispersive laboratory diffractometer should be characterized by a high degree of flexibility and extensibility. By this way the diffractometer can act as a test platform for the realization of different measurement strategies. This applies in particular to the implementation of optical components for guiding the beam path on the primary and the secondary side. Furthermore, the possibility should be considered, to use the diffractometer optionally in the angle dispersive diffraction mode. This opens up further measurement options like the high resolution line profile analysis for example by suppressing fluorescence effects from the material. Figure 1 shows the technical concept of the ED 8-circle diffractometer which has designed in cooperation between the HUBER Diffraktionstechnik GmbH \& Co. KG company, the university Kassel and the HelmholtzZentrum Berlin. This diffractometer will be placed on an exactly aligned table with a granite countertop to realize maximum position stability. The experimental hutch is designed for higher radiation powers, so that in later research activities also X-ray tubes with liquid anodes can be used.

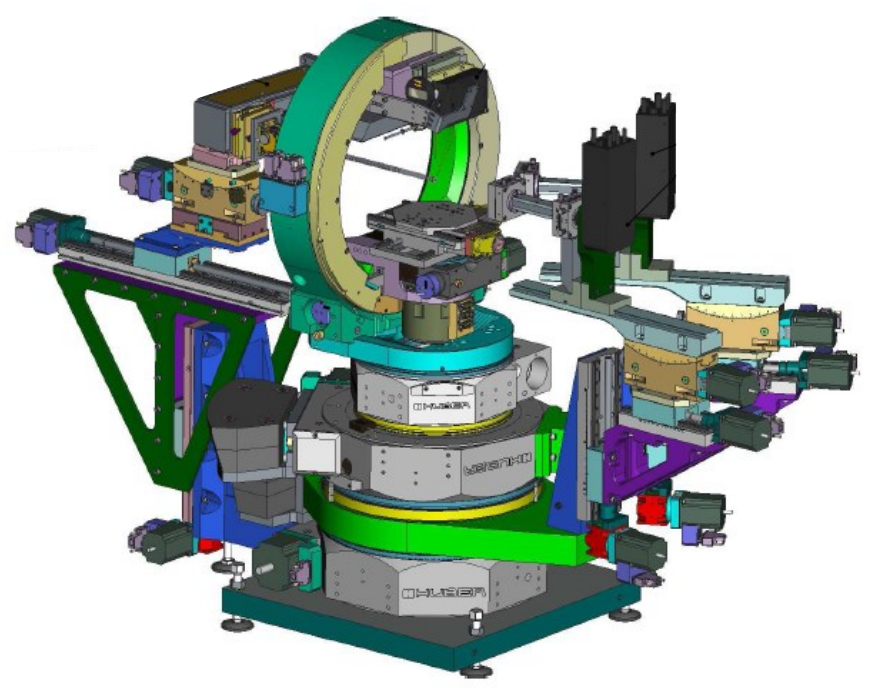

Fig.1: Schematic view of the energy-dispersive laboratory diffractometer with two-detector setup (construction drawing courtesy of HUBER). 
To enable experiments in any diffraction mode and geometry the diffractometer can be operated in the horizontal as well as in the vertical plane. Sample positioning is realized by an Eulerian cradle with integrated $\Phi$-rotation table and $x-y-z$ translation stage. The X-ray tube can be tilted in the vertical plane and translated along the "zero axis" of the diffractometer to allow for flexible distances between the focus and the sample. Both detectors are mounted on separate arms to enable their independent rotation in the horizontal and tilt in the vertical plane. The construction of the two detector arms (see Figure 1) is stiff enough to bear also heavier detector systems, e. g. with a liquid helium reservoir for cooling. The smallest relative angle between the two detector arms, which depends on the lateral detector position and the type of detector is in the range of $16.5^{\circ}$. With an energy resolution of 8,135 eV/channel it is also possible to select some single channels and measure in the angle dispersive mode. By means of optical benches the distance between the sample and the detectors can be varied. Sample alignment is controlled by a Laser/CCD camera system which is mounted in the cradle opposite to the sample. Since it can pivot about the X-axis, correct sample alignment can be controlled even for inclined sample positions.

\section{Optics and detectors}

The main focus of this experimental setup is directed on the X-ray source and the detectors. At synchrotron facilities detectors are in use for energy ranges up to $100 \mathrm{keV}$ or higher and for cooling purposes of these semiconductor detectors a certain amount of liquid nitrogen is needed to be replenished in the cycle of two or three days. The continuous development of the detector electronics allows nowadays to produce semiconductor detectors based on the $\mathrm{Si}(\mathrm{Li}) \mathrm{drift}$ technology, which work reasonably well in the range of 1.5 to $60 \mathrm{keV}$. To keep the design of the instrument as compact as possible we tested the efficiency and energy resolution of such a thermoelectrically cooled $\mathrm{Si}(\mathrm{Li})$ detector which is much smaller concerning its weight as well as dimensions than the rather heavy and big N2(liq.)-cooled solid state Ge detectors. Figure 2 shows that the efficiency of the $\mathrm{Si}(\mathrm{Li})$ detector decreases much stronger for photon energies $>$ about 30 $\mathrm{keV}$ compared to that of the LEGe detector, but is much higher for energies $<30 \mathrm{keV}$.

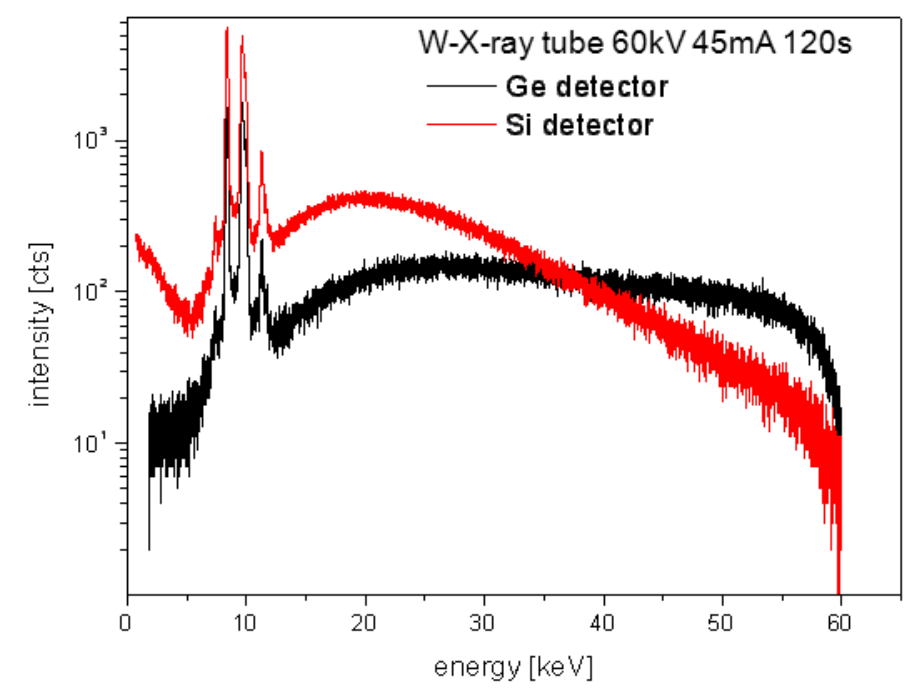

Fig.2: Bremsstrahlung spectrum of a tungsten X-ray tube (long fine focus, operation conditions $60 \mathrm{keV} / 45 \mathrm{~mA}$ ) recorded with a low-energy germanium (LEGe) detector (Canberra, black) and a Si(Li) detector (Baltic Scientific Instruments, red), respectively. The counting time was $120 \mathrm{~s}$ in both cases.

The spot size and the beam divergence of the primary beam are defined by collimators of different length. The equatorial divergence of the diffracted beam is restricted by a $0.15^{\circ}$ soller collimators. To optimize the intensity additionally, it will be also possible to move the source and the detectors in the explained lateral direction to minimize the length of the absorption path through the air. 
Despite of the disadvantage compared to synchrotron facilities caused in the lower flux and the higher divergence, it can be seen from the ED diffraction patterns in Figure 3 that the $\operatorname{Si}(\mathrm{Li})$ detector is able to detect evaluable diffraction lines even at energies close to $60 \mathrm{keV}$ which is the maximum photon energy of the bremsspectrum delivered by a conventional tungsten tube. Moreover, the diagrams indicate that the lower counting efficiency of the $\mathrm{Si}(\mathrm{Li})$ detector for $\mathrm{E}>$ $30 \mathrm{keV}$ is compensated by a higher efficiency for smaller energies. Therefore, it may be expected that one of the preferred fields of application for the $\mathrm{Si}(\mathrm{Li})$ detectors in $\mathrm{ED}$ diffraction could be thin film stress, texture and microstructure analysis, which is usually performed with rather low energy photons.

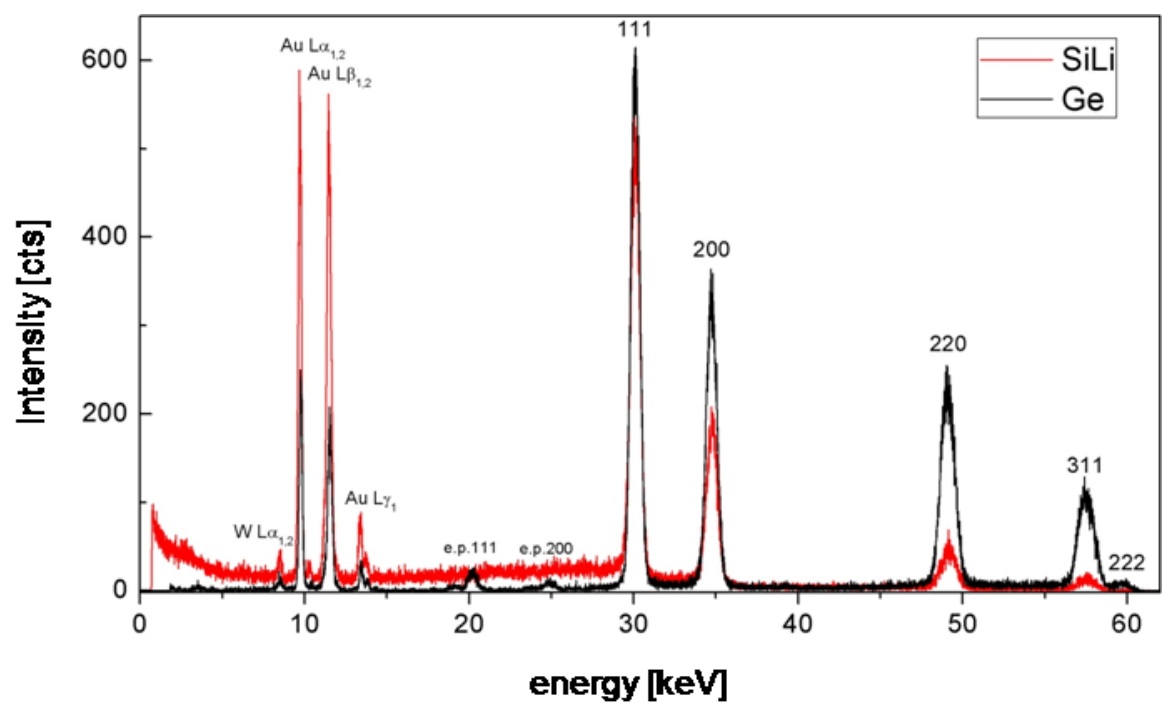

Fig.3: ED diffraction patterns of Au standard powder measured with a LEGe- and a Si(Li)-detector, respectively. The $2 \theta$ angle was $10^{\circ}$, for the operation conditions see Fig. 2.

The resolution $\triangle \mathrm{E} / \mathrm{E}$ of $\mathrm{ED}$ diffraction experiments is controlled by both the geometrical resolution (beam divergence) and the energy resolution of the detector. For the two detectors tested in this paper, it can be realized from Figure 4 that the intrinsic line width is very similar for LEGe and $\mathrm{Si}(\mathrm{Li})$. No geometrically induced line broadening and shift was observed for large tilt angles $\psi$.
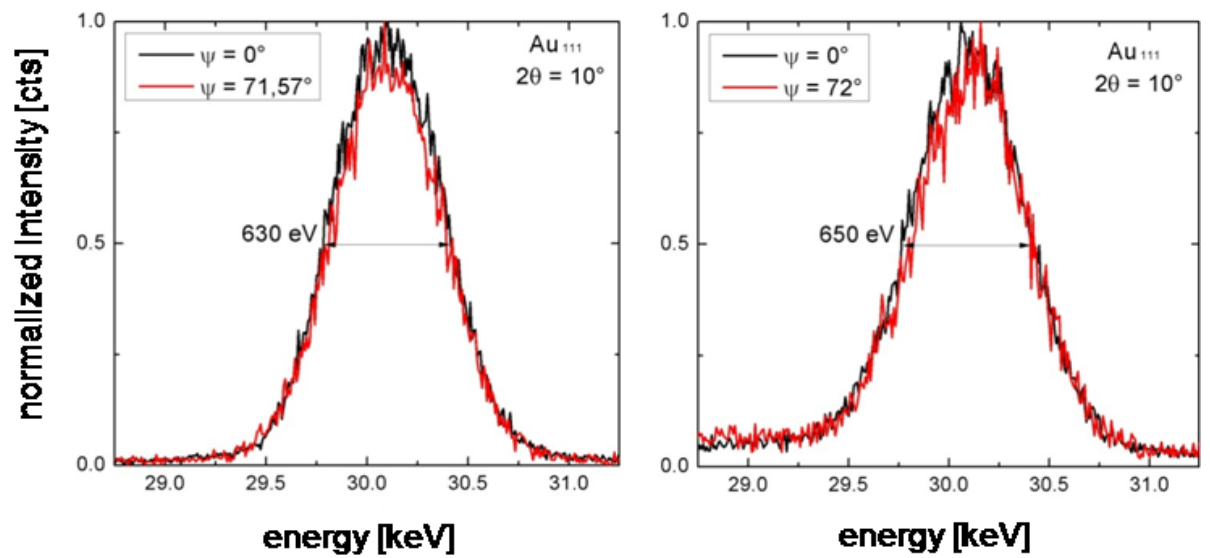

Fig.4: Diffraction line width obtained from $\sin ^{2} \psi$-measurements performed on Au standard powder (cf. Fig. 3). Left: LEGe-detector, right: $\mathrm{Si}(\mathrm{Li})-$ detector. For the purposes of comparison the maximum intensities were normalized. 


\section{Conclusions and outlook}

We have demonstrated that the residual stress analysis by means of energy dispersive diffraction can be also carried out under low photon flux laboratory conditions. Based on this idea the concept for an ED laboratory diffractometer was introduced which will be equipped with two detectors. Recording simultaneously diffraction spectra in two different measuring directions may compensate the main drawback of the significantly longer counting times under laboratory conditions at least partially. In order to fully exploit the features of ED diffraction for depth resolved residual stress analysis under laboratory conditions, new efficient methods for data evaluation have to be worked out. They must take into account, that the number of ED diffraction spectra used for stress depth profiling has to be kept as small as possible to ensure reasonable measuring times. Using the above mentioned 8-circle diffractometer and a tungsten anode any horizontal, vertical, and "mixed" scattering mode can be realized. A main advantage here is that the bremsspektrum of laboratory Xray sources is unpolarized and that compared to synchrotron radiation no intensity losses for $\theta_{\text {hor }}$, $\theta_{\text {hor }}{ }^{\mathrm{D}} \neq 0$ occur. Of crucial importance for the energy resolution in the diffracted spectrums is the careful selection of suitable optical components for beam guiding or beam limiting. According to previous experience, the use of conical collimators on the primary side is recommended, while on the secondary side in this regard, good results were obtained with $0.15^{\circ}$ soller slits [12].

\section{References}

[1] Nagao, M. und Kusumoto, J. Soc. Mater. Sci. Jap. 26 (1977), 576 - 583 (in Japanisch).

[2] Bechtoldt, C. J., Placious, R. C., Boettinger, W. J. und Kuriyama, M. Adv. X-Ray Anal. 25 (1982), $329-338$.

[3] Black, D. R., Bechtoldt, C. J., Placious, R. C. und Kuriyama, M., J. Nondestr. Evaluation 5 (1985), $21-25$.

[4] Wilson, A. J. C., J. Appl. Cryst. 6 (1973), 230 - 237.

[5] Buras, B., Nimura, N. und Olsen, J. S., J. Appl. Cryst. 11 (1978), 137 - 140.

[6] Ruppersberg, H., Detemple, I. und Krier, J., phys. stat. sol. (a) 116 (1989), 681 - 687.

[7] Ruppersberg, H. und Detemple, I., Mat. Sci. Eng. A161 (1993), 41 - 44.

[8] Ruppersberg, H., Adv. X-ray Anal. 37 (1994), 235 - 244.

[9] Stock, C., Dissertation, TU Berlin, 2003 (http://opus.kobv.de/tuberlin/volltexte/2003/636/).

[10] Genzel, Ch., Stock, C. und Reimers, W., Mat. Sci. Eng. A372 (2004), 28 - 43.

[11] Zschenderlein, U., Kämpfe, B., Schultrich, B. und Fritsche, G., Solid State Phenomena 130 (2007), $39-42$.

[12] Genzel, Ch., Krahmer, S., Klaus, M. und Denks, I. A., J. Appl. Cryst. 44 (2011), 1 -12. 